\title{
Cosmological particle creation in conformal gravity
}

\author{
Victor Berezin ${ }^{1, \star}$, Vyacheslav Dokuchaev ${ }^{1,2, \star \star}$, and Yury Eroshenko ${ }^{1, \star \star \star}$ \\ ${ }^{1}$ Institute for Nuclear Research of the Russian Academy of Sciences, Moscow, Russia \\ ${ }^{2}$ National Research Nuclear University MEPhl, Moscow, Russia
}

\begin{abstract}
We constructed the conformally invariant model for scalar particle creation induced by strong gravitational fields. Starting from the "usual" hydrodynamical description of the particle motion written in the Eulerian coordinates we substituted the particle number conservation law (which enters the formalism) by "the particle creation law", proportional to the square of the Weyl tensor (following the famous result by Ya. B. Zel'dovich and A. A. Starobinsky). Then, demanding the conformal invariance of the whole dynamical system, we have got both the (Weyl)-conformal gravity and the Einstein-Hilbert-dilaton gravity action integral. Thus, we obtained something like the induced gravity suggested first by A. D. Sakharov. It is shown that the resulting system is self-consistent. Some future developments of the theory are discussed in the concluding Chapter.
\end{abstract}

\section{Introduction}

The gravitational field causes particle creation by disturbing vacuum fluctuations of scalar, vector, spinor, etc., fields. Owing the results of works by many outstanding scientists [1-6] we know that the renormalization procedure inherent in quantum field theories, requires the adding to the original Einstein-Hilbert gravitational action integral some new terms quadratic in Ricci tensor and scalar curvature (in one loop approximation). The very necessity of these additions supports strongly the idea, first introduced by A. D. Sakharov in 1967 [7] and now known as the induced gravity.

The above mentioned investigations of the particle creation were confined by considering the quantum scalar field on the given background metric (cosmological homogeneous, but anisotropic, space-times). The main obstacle to the accounting for the back reaction is that the rigorous solution of the quantum problem requires knowing the boundary conditions, while the latter can be imposed only after solving the (classical) Einstein equations. Meanwhile the back reaction seems very important because not only the created particles will change the geometry, but the very process of creation, which is purely quantum phenomenon, may violate the well known energy dominance condition and, thus, should be crucial in understanding the global space-time geometry. To avoid this difficulty, we propose to describe the particle creation processes phenomenologically, on the classical level, what should be rather reasonable, when the gravitational fields are strong enough (e. g., in the early universe

\footnotetext{
^e-mail: berezin@inr.ac.ru

$\star \star$ e-mail: dokuchaev@inr.ac.ru

$\star \star \star$ e-mail: eroshenko@inr.ac.ru
} 
and inside black holes). The first attempts to the fulfil such a program proved that, indeed, the energy dominance condition may be violated $[8,9]$.

What would be the "creation law"? Surely, it should be some function of the invariants constructed from the fields responsible for the particle creation. Ya. B. Zel'dovich and A. A. Starobinsky [10] showed that the production rate (e.g., the number of particles created per unit volume per unit time) is proportional to the square of the Weyl tensor, the coefficient being depend on the nature of the particles. In our model we adopt this as the first postulate. It will be shown that this leads us inevitably to the Weyl's gravitational integral. If we neglect all the other possible quadratic combinations of Riemann and Ricci tensors as well as scalar curvature, we will arrive at the so called conformal gravity theory, and, at the same time, confine ourselves to the definite choice of the counterparts, arising from the renormalization of the quantum fields.

Thus, we are almost forced to adopt the second postulate - the conformal invariance of the field theory. In the present paper we consider only the scalar filed, making it responsible for the particle creation. Demanding the conformal invariance we have to add to the "normal" scalar field Lagrangian the term proportional to the curvature scalar, thus reproducing the Einstein-Hilbert dilaton action integral.

The conformal gravity was invented by H. Weyl in 1918 [11]. His motivation was to construct the unified theory of two (known at the time) fundamental fields: electromagnetic and gravitational ones. Since the electromagnetic field ("identified with the Maxwell equations") is invariant under the conformal transformations, H. Weyl proposed the conformal invariant Lagrangian for the gravitational field. Then. it was recognized that the Weyl's gravity allows only massless particles to exist. On this ground the theory was rejected by $\mathrm{H}$. Weyl himself and by A. Einstein. But, nowadays, this unpleasant feature can be "corrected" by Braut-Englert-Higgs mechanism for the spontaneous symmetry breaking [12]. The vacuum space-time with very high symmetry is a good candidate for the creation of the universe from "nothing" [13]. It can be easily verified that all the homogeneous isotropic space-times have zero Weyl tensor. So. they automatically solve the vacuum equations of the conformal gravity. The idea that the initial state of the universe should be conformal invariant is advocated also by R. Penrose [14, 15] and G. 't Hooft [16].

This paper is devoted to the detailed description of our model for particle creation in the conformal gravity. We will use, in particular, the formalism from our previous papers [17, 18] on the conformal gravity.

Throughout the paper we use the units $\hbar=c=1$ and the sign convention as in [19], i.e., the signature of the metric tensor is $g_{\mu \nu}$ is $(+,-,-,-)$, the Riemann curvature tensor is defined as

$$
R_{\nu \lambda \sigma}^{\mu}=\frac{\partial \Gamma_{v \sigma}^{\mu}}{\partial x^{\lambda}}-\frac{\partial \Gamma_{v \lambda}^{\mu}}{\partial x^{\sigma}}+\Gamma_{\chi \lambda}^{\mu} \Gamma_{v \sigma}^{\varkappa}-\Gamma_{\varkappa \sigma}^{\mu} \Gamma_{v \lambda}^{\varkappa}
$$

while the Ricci tensor is the following convolution

$$
R_{v \sigma}=R_{\nu \mu \sigma}^{\mu} .
$$

The scalar curvature is $R=g^{v \sigma} R_{\nu \sigma}$, and $\Gamma_{\mu \nu}^{\lambda}$ are the metric connections, i.e., the covariant derivatives of the metric tensor are zero.

\section{Phenomenological model}

\subsection{Hydrodynamics with varying particle number}

We start with construction of the hydrodynamical part of our model. In the "classical" hydrodynamics there exist two different sets of dynamical variables, the so called Lagrangian and Eulerian coordinates. The first of them are comoving, i.e., the observer is sitting on some world-line. So, using the 
least action principle, one has to vary the trajectory of the (quasi)-particles. Since in such a case we cannot take into account the very processes of both creation and annihilation of particles (i.e., trajectories), it is not appropriate for our purposes. Therefore, we need to use the Eulerian description, when the dynamical variables are fields, namely, the particle number density $n(x 0)$ and the four-velocities. The action integral in this case is [20]

$$
S_{\text {hydro }}=-\int \varepsilon(X, n) \sqrt{-g} d x+\int \lambda_{0}\left(u^{\mu} u_{\mu}-1\right) \sqrt{-g} d x+\int \lambda_{2} X_{, \mu} u^{\mu} \sqrt{-g} d x+\int \lambda_{1}\left(n u^{\mu}\right)_{; \mu} \sqrt{-g} d x,
$$

where $\varepsilon(X, n)$ is the invariant energy density, $n(x)$ - invariant particle number density, $u^{\mu}(x)$ four-velocity of the particle flow, $X(x)$ is the auxiliary dynamical variable introduced in order to avoid the identically zero vorticity of particle flow. It enters the action integral with the Lagrange multiplier $\lambda_{2}$, indicating the constraint $X_{, \mu} u^{\mu}=0$, i.e., $X(x)=$ const on the trajectories, thus enumerating them. The other two Lagrange multipliers, $\lambda_{0}(x)$ and $\lambda_{1}(x)$ are responsible, respectively, for the constraints $u^{\mu} u_{\mu}=1$ (natural normalization of the four-velocities) and $\left(n u^{\mu}\right)_{; \mu}=0$ - particle number conservation law. The semicolon ";" denotes a covariant derivative with respect to the metric $g_{\mu \nu}$.

Our aim is to incorporate into the formalism the particle "creation law"

$$
\left(n u^{\mu}\right)_{; \mu}=\Phi(i n v) \neq 0 .
$$

Evidently, the function $\Phi$ should depend on some invariants of the fields causing this particle creation. Here we would like to explore the fundamental result by Ya. B. Zel'dovich and A. A. Starobinsky [10] obtain for the cosmological particle production

$$
\left(n u^{\mu}\right)_{; \mu}=\beta C^{2} .
$$

where $C^{2}$ is the square of the Weyl tensor $C^{\mu}{ }_{\nu \lambda \sigma}$ (its definition as well as some most important properties see e. g., in $[17,18])$ and the coefficient $\beta$ depends on the type of particles under consideration. We will consider this "creation law" as our first postulate. So, the hydrodynamical part of the action integral now becomes

$$
S_{\text {hydro }}=-\int \varepsilon(X, n) \sqrt{-g} d x+\int \lambda_{0}\left(u^{\mu} u_{\mu}-1\right) \sqrt{-g} d x+\int \lambda_{2} X_{, \mu} u^{\mu} \sqrt{-g} d x+\int \lambda_{1}\left(\left(n u^{\mu}\right)_{; \mu}-\beta C^{2}\right) \sqrt{-g} d x,
$$

Very important note. The Lagrange multiplier $\lambda_{1}$ is, actually, defined up to the additive constant. Indeed, let us replace $\lambda_{1} \rightarrow \lambda_{1}+\gamma_{0}, \gamma_{0}=$ const, then

$$
\gamma_{0} \int\left(\left(n u^{\mu}\right)_{; \mu}-\beta C^{2}\right) \sqrt{-g} d x=\gamma_{0} \int\left(\left(n \sqrt{-g} u^{\mu}\right)_{, \mu}-\beta C^{2} \sqrt{-g}\right) d x
$$

Due to the identity $\left(n u^{\mu}\right)_{; \mu} \sqrt{-g}=\left(n \sqrt{-g} u^{\mu}\right)_{, \mu}$, the corresponding volume integral transforms into the surface integral with no effect on the dynamical equations. Thus, we are left with the same "creation law" as before plus the Weyl gravitational action

$$
S_{\mathrm{grav}}^{\mathrm{Weyl}}=-\gamma_{0} \beta \int C^{2} \sqrt{-g} d x
$$

Thus, the conformal gravity is intrinsically contained in our hydrodynamical part of the total action integral, prior to the introducing the gravitational action itself! 


\subsection{Scalar field and conformal invariance}

By the conformal transformation we will understand the space-time dependent scaling of the metric tensor $g_{\mu \nu}$,

$$
d s^{2}=g_{\mu \nu}(x) d x^{\mu} d x^{\nu}=\Omega^{2} \hat{g}_{\mu \nu}(x) d x^{\mu} d x^{\nu}=\Omega^{2}(x) \hat{d} s^{2} .
$$

The conformal invariance means

$$
\frac{\delta S_{\text {tot }}}{\delta \Omega}=0 .
$$

Therefore, we can (and will) consider the conformal factor $\Omega$ as a dynamical variable and make variations independently in $\Omega$ and in $\hat{g}_{\mu v}$ [16].

Let

$$
S_{\text {tot }}=S_{\text {grav }}+S_{\text {matter }}
$$

By definition

$$
\delta S_{\text {matter }}=\frac{1}{2} \int T_{\mu \nu} \sqrt{-g} \delta g^{\mu v} d x \quad \text { and } \quad \delta S_{\text {matter }}=\frac{1}{2} \int \hat{T}_{\mu v} \sqrt{-\hat{g}} \delta \hat{g}^{\mu v} d x
$$

where $T_{\mu \nu}\left(\hat{T}_{\mu \nu}\right)$ is the matter energy-momentum tensor. Consider, first, the following transformation of the metric tensor

$$
\delta g^{\mu v}=-\frac{2}{\Omega^{3}} \hat{g}^{\mu v} \delta \Omega=-\frac{2}{\Omega} g^{\mu v} \delta \Omega .
$$

Suppose

$$
\frac{\delta S_{\text {grav }}}{\delta \Omega}=0
$$

then

$$
0=\delta S_{\text {matter }}=-\int T_{\mu \nu} g^{\mu \nu} \frac{\delta \Omega}{\Omega} \sqrt{-g} d x,
$$

that is, the trace of the energy-momentum tensor should be zero:

$$
\operatorname{Tr}\left(T_{\mu \nu}\right)=\operatorname{Tr}\left(\hat{T}_{\mu \nu}\right)=0 .
$$

If one considers the metric tensor transformation of the kind

$$
\delta g^{\mu \nu}=\Omega^{2} \delta \hat{g}^{\mu \nu},
$$

then, as can be easily seen,

$$
\hat{T}_{\mu \nu}=\Omega^{2} T_{\mu \nu} \quad\left(\hat{T}_{v}^{\mu}=\Omega^{4} T_{v}^{\mu}, \quad \hat{T}^{\mu \nu}=\Omega^{6} T^{\mu \nu}\right) .
$$

Let us go further on. The question arises: quanta of what kind a field are creating? The most simple choice is the scalar field. And the simplest action integral is

$$
S_{\text {scalar }}=\int\left(\frac{1}{2} \chi^{\mu} \chi_{\mu}-\frac{1}{2} m^{2} \chi^{2}\right) \sqrt{-g} d x .
$$

Here $\chi_{\mu}=\chi_{, \mu}$ (comma denotes the partial derivative), $\chi^{\mu}=g^{\mu \nu} \chi_{v}$ and $m$ is some constant with the dimension of mass. After the "standard" conformal transformations, namely

$$
g^{\mu \nu}=\Omega^{2} \hat{g}^{\mu \nu}, \quad \chi=\frac{1}{\Omega} \hat{\chi},
$$


one gets

$$
S_{\text {scalar }}=\int\left(\frac{1}{2} \hat{\chi}^{\mu} \hat{\chi}_{\mu}-\frac{1}{\Omega} \hat{\chi}_{\mu} \Omega^{\mu}+\frac{1}{2} \frac{\hat{\chi}^{2}}{\Omega^{2}} \Omega_{\mu} \Omega^{\mu}-\frac{1}{2} m^{2} \Omega^{2} \hat{\chi}^{2}\right) \sqrt{-\hat{g}} d x .
$$

Now indices are raising and lowering with the metric $\hat{g}_{\mu v}\left(\hat{g}^{\mu v}\right)$. How to make this action conformally covariant? The recipe is well known: one should add into the Lagrangian the term $(R / 12) \chi^{2}$, where $R$ is the scalar curvature, constructing from the metric $g_{\mu v}$. The result is

$$
\begin{aligned}
S_{\text {scalar }} & =\int\left(\frac{1}{2} \chi^{\mu} \chi_{\mu}+\frac{R}{12} \chi^{2}-\frac{1}{2} m^{2} \chi^{2}\right) \sqrt{-g} d x \\
& =\int\left(\frac{1}{2} \hat{\chi}^{\mu} \hat{\chi}_{\mu}+\frac{\hat{R}}{12} \hat{\chi}^{2}-\frac{1}{2} m^{2} \Omega^{2} \hat{\chi}^{2}\right) \sqrt{-\hat{g}} d x-\frac{1}{2} \int\left(\hat{\chi}^{2} \frac{\Omega^{\lambda}}{\Omega}\right)_{\mid \lambda} \sqrt{-\hat{g}} d x
\end{aligned}
$$

Here the vertical line "l" denotes the covariant derivative with respect to the metric $\hat{g}_{\mu \nu}$. The last term can be transformed to the surface integral, it does not effect the dynamics. Remarkably enough, that started with no gravitational action at all, we have got now both the conformal gravity (as a part of the "creation law") and the Einstein-Hilbert-dilaton gravity (as a part of the conformally covariant scalar field Lagrangian).

It seems that if one puts $m=0$, everything else will be all right. But, it is not so easy, there exists a problem [16]. This problem concerns the signs. With the "correct" sign for the kinetic term $(1 / 2) \chi^{\mu} \chi_{v}$, we have the "wrong" sign for the Einstein-Hilbert-dilaton part, $+(1 / 12) \hat{R} \hat{\chi}^{2}$ (with our sign convention there should be "-" instead of "+"), and vice-versa. Our choice is the "correct" sign for $\hat{R}$, i. e., $-(1 / 12) \hat{R} \hat{\chi}^{2}$, and the "wrong" sign for the kinetic term, i. e., $-(1 / 2) \chi^{\mu} \chi_{v}$. This requires some explanation. First of all, we do not care about the "correct" sign for the kinetic term, because our scalar $\chi$ is not the "genuine" (i. e., fundamental) one. Some part of it we have already "used" as the created particles. The residual part can be viewed as the vacuum fluctuations that consist of virtual particles, including the conformal anomaly, which is responsible for the creation process. Moreover, the "wrong" sign in the kinetic term means the absence of the lower bound for the energy and allows even infinite number of the created particles (let us remember the C-field in the "steady state" cosmological model by F. Hoyle and J. V. Narlikar [21]). Besides, we are not going to consider our field $\chi$ as an independent dynamical variable. One more thing. If the scalar field $\chi$ is an independent dynamical variable, then, why it "knows" about the conformal transformation $g_{\mu \nu}=\Omega^{2} \hat{g}_{\mu \nu}$ and adjusts itself properly, 1. e., $\hat{\chi}=\Omega \chi$ ? Only, when this field is a part of it! Fortunately, in our case it is not so, and one can always choose the conformal factor, $\Omega=\varphi$, in such a way that

$$
\hat{\chi}=\frac{1}{\ell} \varphi
$$

where $\ell$ is some factor having dimension of length (it is introduced in order to keep the action integral dimensionless). Then, the action integral for the scalar field takes the form

$$
S_{\text {scalar }}=-\frac{1}{\ell^{2}} \int\left(\frac{1}{2} \varphi^{\mu} \varphi_{\mu}+\frac{\hat{R}}{12} \varphi^{2}-\frac{1}{2 \ell^{2}} m^{2} \varphi^{4}\right) \sqrt{-\hat{g}} d x
$$

There appears the self-interaction term, $\varphi^{4}$. It must be noted that the power 4 in this term is only in the case of the four-dimensional space-time (it depends on the space-time dimensions). Here, two comments are in order. First, the above action is covariant under the conformal transformation, $\varphi($ new $)=\hat{\Omega} \varphi($ old $), g_{\mu \nu}($ old $)=\hat{\Omega}^{2} g_{\mu \nu}($ new $), \sqrt{-g}($ old $)=\hat{\Omega} \sqrt{-g(\text { new })}$, what can be easily checked. Second, it is now evident, that $3 m^{2}=\Lambda$ plays the role of the (bare) cosmological term. 
To finish this Subsection we write down the energy-momentum tensor $T_{\mu \nu}$ for our (new) scalar field $\varphi$, obtained by varying $S_{\text {scalar }}$ in $\hat{g}_{\mu \nu}$ :

$$
\begin{aligned}
\hat{T}_{\mu \nu}^{\text {scalar }}= & -\frac{1}{\ell^{2}} \varphi_{\mu} \varphi_{\mu}+\frac{1}{2 \ell^{2}} \varphi^{\sigma} \varphi_{\sigma} \hat{g}_{\mu \nu}-\frac{1}{2 \ell^{2}} m^{2} \varphi^{4} \hat{g}_{\mu \nu} \\
& -\frac{1}{6 \ell^{2}}\left(\varphi^{2}\left(\hat{R}_{\mu \nu}-\frac{1}{2} \hat{g}_{\mu \nu} \hat{R}\right)-2\left(\left(\varphi \varphi_{v}\right)_{\mid \mu}-\left(\varphi \varphi^{\sigma}\right)_{\mid \sigma} \hat{g}_{\mu \nu}\right)\right)
\end{aligned}
$$

Note the appearance of the second derivatives. The trace of this tensor equals

$$
\operatorname{Tr}\left(\hat{T}_{\mu \nu}^{\text {scalar }}\right)=-\frac{1}{\ell^{2}}\left(\varphi \varphi_{\mid \sigma}^{\sigma}-\frac{\hat{R}}{6} \varphi^{2}+2 m^{2} \varphi^{4}\right) .
$$

\subsection{Hydrodynamics and conformal covariance}

Since we consider now the conformal factor $\varphi$ and transformed metric tensor $\hat{g}_{\mu v}$ as the independent dynamical variables, the above written hydrodynamical action integral should be properly "updated". Let us start with analysing the "creation law",

$$
0=\left(\left(n u^{\mu}\right)_{; \mu}-\beta C^{2}\right) \sqrt{-g}=\left(\left(n \sqrt{-g} u^{\mu}\right)_{, \mu}-\beta C^{2} \sqrt{-g}\right) .
$$

It is well known that in the four-dimensional space-time the combination $C^{2} \sqrt{-g}$ is invariant under conformal transformation, i. e.,

$$
C^{2} \sqrt{-g}=\hat{C}^{2} \sqrt{-\hat{g}}
$$

So should be the full derivative $\left(n u^{\mu} \sqrt{-g}\right)_{, \mu}$. The square of the interval $d s^{2}$ transforms as

$$
d s^{2}=g_{\mu \nu} d x^{\mu} d x^{\nu}=\varphi^{2} \hat{g}_{\mu \nu} d x^{\mu} d x^{\nu}=\varphi^{2} d \hat{s}^{2},
$$

therefore, the four-velocity $u^{\mu}$ behaves as

$$
u^{\mu}=\frac{d x^{\mu}}{d s}=\frac{1}{\varphi} \frac{d x^{\mu}}{d \hat{s}}=\frac{1}{\varphi} \hat{u}^{\mu},
$$

and, respectively,

$$
u_{\mu}=g_{\mu \nu} u^{v}=\varphi \hat{g}_{\mu \nu} \hat{u}^{v}=\varphi \hat{u}_{\mu}
$$

Thus

$$
n \sqrt{-g} u^{\mu}=n \varphi^{3} \sqrt{-\hat{g}} \hat{u}^{\mu}=\hat{n} \hat{u}^{\mu},
$$

where we introduced the new notation

$$
\hat{n}=n \varphi^{3} \sqrt{-\hat{g}} .
$$

It is clear that in the comoving coordinate system $\hat{n}$ is nothing but the particle number per unit spatial coordinate volume and, thus, the conformally invariant quantity. So, the "creation law" does not contain now the conformal factor $\varphi$ explicitly. Therefore, the hydrodynamical part of the total action integral becomes

$$
\begin{aligned}
S_{\text {hydro }}= & -\int \varepsilon\left(X, \frac{\hat{n}}{\varphi^{3} \sqrt{-\hat{g}}}\right) \varphi^{4} \sqrt{-\hat{g}} d x+\int \lambda_{0}\left(\hat{u}^{\mu} \hat{u}_{\mu}-1\right) \varphi^{4} \sqrt{-\hat{g}} d x \\
& +\int \lambda_{2} X_{, \mu} \hat{u}^{\mu} \varphi^{3} \sqrt{-\hat{g}} d x+\int \lambda_{1}\left(\left(\hat{n} \hat{u}^{\mu}\right)_{, \mu}-\beta \hat{C}^{2} \sqrt{-\hat{g}}\right) d x,
\end{aligned}
$$


and now the hydrodynamical variables are $\hat{n}, \hat{u}^{\mu}$ and $X$. Let us write down the corresponding equations of motion

$$
\begin{aligned}
& \frac{\delta S_{\text {hydro }}}{\delta \hat{n}}=-\frac{\partial \epsilon}{\partial n} \frac{1}{\varphi^{3} \sqrt{-\hat{g}}}-\lambda_{1, \sigma} \hat{u}^{\sigma}=0, \\
& \frac{\delta S_{\text {hydro }}}{\delta \hat{u}^{\mu}}=2 \lambda_{0} \hat{u}_{\mu} \varphi^{4}+\lambda_{2} \varphi^{3} X_{, \mu}-\lambda_{1, \mu} \frac{\hat{n}}{\sqrt{-\hat{g}}}=0, \\
& \frac{\delta S_{\text {hydro }}}{\delta X}=-\frac{\partial \epsilon}{\partial X} \varphi^{4}-\frac{\left(\lambda_{2} \varphi^{3} \sqrt{-\hat{g}} \hat{u}^{\sigma}\right)_{, \sigma}}{\sqrt{-\hat{g}}}=0 .
\end{aligned}
$$

To these we should add, of course, the constraints that follow from variation of the action integral in Lagrange multipliers $\lambda_{0}, \lambda_{1}$ and $\lambda_{2}$ :

$$
\hat{u}^{\sigma} \hat{u}_{\sigma}=u^{\sigma} u_{\sigma}=1, \quad X_{\sigma} \hat{u}^{\sigma}=X_{\sigma} u^{\sigma}=0, \quad\left(\hat{n} \hat{u}^{\mu}\right)_{, \mu}=\beta \hat{C}^{2} \sqrt{-\hat{g}},
$$

the last of them being equivalent to $\left(n u^{\mu}\right)_{; \mu}=\beta C^{2}$. The above equations of motion can be also written in terms of the quantities without "hats", namely

$$
-\frac{\partial \epsilon}{\partial n}-\lambda_{1, \sigma} u^{\sigma}=0, \quad 2 \lambda_{0} u_{\mu}+\lambda_{2} X_{, \mu}-n \lambda_{1, \mu}=0, \quad-\frac{\partial \epsilon}{\partial X}-\left(\lambda_{2} u^{\sigma}\right)_{, \sigma}=0 .
$$

It is not difficult to extract the Lagrange multiplier $\lambda_{0}$ from these equations. Indeed, by making the convolution of the second of the equations with the four-velocity vector $u^{\mu}$ and using the constraints, we get, after comparing the results with the first of the equations, that

$$
2 \lambda_{0}=-n \frac{\partial \epsilon}{\partial n}
$$

Then, introducing the pressure $p$ in the usual way, $p=-\epsilon+n \frac{\partial \epsilon}{\partial n}$, one obtains

$$
2 \lambda_{0}=-(\epsilon+p) \text {. }
$$

The next step is to compute the hydrodynamical part of the total energy-momentum tensor. Omitting the details, we present here the result:

$$
\begin{aligned}
\hat{T}_{\mu \nu}^{\text {hydro }}= & -\frac{\hat{n}}{\varphi^{3} \sqrt{-\hat{g}}} \frac{\partial \epsilon}{\partial n} \hat{g}_{\mu \nu} \varphi^{4}+\epsilon \varphi^{4} \hat{g}_{\mu \nu}-2 \lambda_{0} \varphi^{4} \hat{u}^{\mu} \hat{u}^{\nu}-\lambda_{0}\left(\hat{u}^{\sigma} \hat{u}_{\sigma}-1\right) \varphi^{4} \hat{g}_{\mu \nu}-\lambda_{2} X_{, \sigma} \hat{u}^{\sigma} \varphi^{3} \hat{g}_{\mu \nu} \\
& -4 \beta \varphi^{2}\left(\left(\lambda_{1} \hat{C}_{\mu \sigma \nu \lambda}\right)^{|\lambda| \sigma}+\frac{1}{2} \lambda_{1} \hat{C}_{\mu \lambda \nu \sigma} \hat{R}^{\lambda \sigma}\right) .
\end{aligned}
$$

or

$$
\hat{T}_{\mu \nu}^{\text {hydro }}=(\varepsilon+p) \varphi^{4} \hat{u}_{\mu} \hat{u}_{\nu}-p \varphi^{4} \hat{g}_{\mu \nu}-4 \beta \varphi^{2}\left(\left(\lambda_{1} \hat{C}_{\mu \sigma \nu \lambda}\right)^{|\lambda| \sigma}+\frac{1}{2} \lambda_{1} \hat{C}_{\mu \lambda \nu \sigma} \hat{R}^{\lambda \sigma}\right) .
$$

with the trace, equals to

$$
\operatorname{Tr}\left(T_{\mu \nu}^{\text {hydro }}\right)=(\varepsilon-3 p) \varphi^{4}
$$

The total trace equals

$$
\operatorname{Tr}\left(T_{\mu \nu}^{\mathrm{tot}}\right)=-\frac{1}{\ell^{2}}\left(\varphi \varphi_{\mid \sigma}^{\sigma}-\frac{\hat{R}}{6} \varphi^{2}+2 m^{2} \varphi^{4}\right)+(\varepsilon-3 p) \varphi^{4}
$$


Finally, let us write the result of the variation of the total action integral in $\varphi$, which can be considered as one of the equations of motion as well as the consequence of the postulated conformal invariance. One gets

$$
\frac{1}{\ell^{2}}\left(\varphi_{\mid \sigma}^{\sigma}-\frac{1}{6} \hat{R} \varphi+2 m^{2} \varphi^{3}\right)+(\varepsilon-3 p) \varphi^{3}=0,
$$

as it should be: $\operatorname{Tr}\left(T_{\mu \nu}^{\text {tot }}\right)=0$ ! The relation $\hat{T}_{\mu \nu}=\varphi T_{\mu \nu}$ can be also easily verified. This proves the self-consistency of our model. Note that in no way $\varphi$ can to be zero value, since this would lead to to the degeneracy of the whole space-time.

\section{Conclusion and Discussion}

\section{A. Conclusion}

We have constructed the self-consistent conformally invariant phenomenological model for particle creation in the presence of strong gravitational fields. The word "phenomenological" means that we adopted classical description both for the created particles (hydrodynamics) and for the "creation law".

This "creation law" enters the action integral with the corresponding Lagrange multiplier and substitute the particle number conservation law in the conventional hydrodynamics. The idea (and our hope) is that such an inclusion of the particle creation law straight into the least action formalism will cause the essential change in the structure of the energy-momentum tensor and will lead to the violation of the energy dominance condition and, thus, will take into account (to some extent) the quantum character of the particle creation process. This idea is not quite new, it was already explored by one of the authors. The new thing is combining of the method with the postulated conformal invariance of the whole theory. This allows to restrict the possible functional form of the "creation law" up to the square of the Weyl tensor.

It appeared, to our surprise, that the above mentioned Lagrange multiplier can be determined only uo to an arbitrary constant. This means that the Weyl gravitational action is, actually, already incorporated into the formalism and does not need to be introduced artificially. The local conformal invariance, taken as the fundamental symmetry, has one more important consequence. In order to make it possible to create particles we need some fields which quanta are these very particles. The simplest is the scalar field. One needs it also because it is the scalar self-interacting field that gives the masses to particles through the Brout-Englert-Higgs mechanism (which also makes the conformal gravity meaningful). If one uses the simplest (again!) form for the scalar field Lagrangian, i.e., "the kinetic term + the mass term", then, in order to make it conformally covariant, it is necessary to introduce also the term proportional to the scalar curvature. Therefore, starting from hydrodynamics, needed for the description of the created particles and introducing the conformally invariant creation law plus the conformally covariant scalar field Lagrangian, we arrived at the conformal gravity theory with the Weyl Lagrangian plus the Einstein-Hilbert-dilaton gravity. This supports the idea, first discussed by A. D. Sakharov, about the induced gravity.

One more thing. In order to have the "correct" sign for the scalar curvature one has to choose the "wrong" sign for the kinetic term in the scalar field Lagrangian. But this causes no conceptual difficulties at all, since our scalar field is not the genuine (fundamental), it is simply the "vacuum residual" part of some entity, the "above-vacuum" part of which is already present in the form of the created particles, and its "conformal anomalous" part is already included into the "creation law". Therefore, it is very "natural" to identify the "vacuum residual" part with the conformal factor of the metric tensor. The mass term now plays the twofold role, it produces the self-interaction and the cosmological term, both initially absent. 


\section{B. Discussion}

1. The model presented above, is very minimalistic. The matter is not only in that we did not include into consideration the electromagnetic (abelian) and other (nonabelian) gauge fields, causing creation of the particle-antiparticle pairs with opposite charges. Here we restricted ourselves by the specific form of the "creation law", when the rate of particle production is proportional to the square of the Weyl tensor. The absence of other possible terms may be explained by the adopted conformal invariance principle. Indeed, $C^{2} \sqrt{-g}$ is conformally invariant. It is usually claimed that the latter is the only conformally invariant combination quadratic in Riemann curvature tensor in four dimensions. But, there exists yet another quadratic conformally invariant combination, namely, the socalled Hirzebruch-Pontryagin density $R^{\mu \nu \lambda \sigma *} R_{\mu \nu \lambda \sigma} \sqrt{-g}$, where "star" means that ${ }^{*} R_{\mu \nu \lambda \sigma}=\epsilon_{\mu \nu \alpha \beta} R_{\lambda \sigma}^{\alpha \beta}$. It is the total derivative and, therefore, when in the action integral, does not alter the equations of motion. In our model, however, it would enter together with the Lagrange multiplier $\left(\lambda_{1}\right)$ and would have an influence on the whole situation. Of course, it is not a genuine scalar, but pseudoscalar. And may be be, it is good, manifesting the $T$-violation in the irreversible particle creation processes.

2. Actually, our gravitational equations, when written in terms of quantities without "hats", look as

$$
R_{\mu \nu}-\frac{1}{2} g_{\mu \nu} R-\Lambda g_{\mu \nu}=6 l^{2} T_{\mu \nu}^{\mathrm{hydro}}
$$

( $\Lambda$ is the cosmological constant). Therefore this is just Einstein gravity, but with the modified energymomentum tensor, which includes the terms responsible for the particle creation. Nevertheless, the whole theory is conformally invariant on the action integral level, due to the special choice of the dynamical variables. From this it follows immediately that the only solution to the spherically symmetric vacuum equations, free of singularities and stable against the particle creation, is the (anti)-de Sitter or Minkowski space-time. (In the case of spherical symmetry, the vanishing $C^{2}$ implies the vanishing $C$ itself, and this singles out the above mentioned metrics.) It sounds not good because the absence of the observers (no particles at all!) would seem to be in favour of the conformally invariant vacuum state. Remember that the existence of such a vacuum was one of the main motivation to reconsider the conformal gravity after the decades of disregarding.

There exists, however, the possibility (may be, exotic one) to switch on the particle creation and still have a vacuum state. To reach this goal, let us allow to create particles of both positive and negative energies (in equal amounts). This resembles the Dirac's sea. Then, we would be much less restrictive in constructing, the already dynamical, vacuum solution. Possible quantum fluctuations will have either positive or negative energies. Those with negative energies will be spreading away due to the self-anti-gravity, while that ones with positive energies will be subject to the usual gravitational instability.

3. The most important problem is how to organize the Braut-Englert-Higgs mechanism for generating particle's rest masses. The conventional line of reasoning is inapplicable here, because the usual (and the most convenient) solution $\varphi=0$ is impossible in our scheme, since it would mean the conformal factor vanishes and, thus, the very notion if the metrical space-time would become meaningless.

4. Let us imagine that the above problem is already solved. Then, we are able to construct the observers equipped with the clocks and other measurement devices, engines for correcting trajectories and all that. In the self-consistent theory these observers cannot be arbitrary at all. For example, the so called vacuum observers (those, "sitting" outside the matter distribution) should see (= measure) different things, depending on their trajectories: the uniformly accelerated ones must be surrounded by the thermal bath, having the Unruh temperature. The simplest construction of this kind (but without the thermal bath) was done in [22]. 
5. All these problems are for the future investigations.

\section{Acknowledgments}

The reported study was partially supported by RFBR, research project No. 15-02-05038 a. The authors would like to thank Alexey Smirnov for numerous discussions and comments. One of us (V. B) is also grateful to Artyom Starodubtsev, Michail Smolyakov and Igor Gulamov for helpful discussions.

\section{References}

[1] L. Parker, Phys. Rev., 183, 1057-1068 (1969)

[2] A. A. Grib and S. G. Mamaev, Sov. J. Nucl. Phys., 10, 722-725 (1970)

[3] V. A. Berezin, V. A. Kuzmin and I. I. Tkachev, Phys. Lett. B, 124, 479-483 (1983)

[4] Ya. B. Zel'dovich, JETP Lett., 9, 307-312 (1970)

[5] Ya. B. Zel'dovich and A. A. Starobinskii, Sov. Phys. — JETP, 34, 1159-1165 (1972)

[6] V. N. Lukash, A. A. Starobinskii, Sov. Phys. — JETP, 39, 742-747 (1974)

[7] A. D. Sakharov, Sov. Phys. Doklady, 12, 1040-1044 (1968)

[8] V. A. Berezin, Intern, J. Mod. Phys. A, 2, 1591-1615 (1987)

[9] V. A. Berezin, Proc. 15th Russian Grav. Conf. "RUSGRAV-15”, Jun. 30 - Jul. 05, Kazan, Russia, 58-62 (2014); arXiv:1404.3582 [gr-qc]

[10] Ya. B. Zel'dovich and A. A. Starobinskii, JETP Lett., 26, 252-255 (1977)

[11] H. Weyl, Mathem. Zeit., 2, 384-411 (1918)

[12] G. 't Hooft, arXiv:1410.6675 [gr-qc].

[13] A. Vilenkin, Phys. Lett. B, 117, 25-28 (1982)

[14] R. Penrose, Cycles of time: An extraordinary new view of the Universe (The Random House, London, 2010)

[15] R. Penrose, Found. Phys, 44, 873-890 (2014)

[16] G. 't Hooft, arXiv:1511.04427 [gr-qc]

[17] V. A. Berezin, V. I. Dokuchaev, and Yu. N. Eroshenko, JCAP 2016, 019 (2016); arXiv:1412.2917v1 [gr-qc]

[18] V. A. Berezin, V. I. Dokuchaev, and Yu. N. Eroshenko, J. Mod. Phys. A, 31, 1641004 (2016); arXiv:1509.00212 [gr-qc]

[19] L. D. Landau and E. M. Lifsitz, The classical theory of fields, "Course of theoretical physics, vol 2. Forth edition (Butterworth-Heinemann, Oxford, 2000)

[20] J. R. Ray, J. Math. Phys. 13, 1451-1453 (1972)

[21] F. Hoyle and J. V. Narlikar, Proc. Roy. Soc. London. A, 273, 1-11, (1963)

[22] V. A. Berezin, arXiv:1106.0670 [gr-qc] 